\title{
Short-term Results of Microendoscopic Muscle- preserving Interlaminar Decompression versus Spinal Process Splitting Laminectomy
}

\author{
Ryunosuke Fukushi $^{1}$ Mitsunori Yoshimoto ${ }^{1}$ Noriyuki lesato $^{1}$ Yoshinori Terashima ${ }^{1}$ \\ Tsuneo Takebayashi ${ }^{1}$ Toshihiko Yamashita ${ }^{1}$
}

${ }^{1}$ Department of Orthopaedic Surgery, Sapporo Medical University, Sapporo, Japan

J Neurol Surg A 2018;79:511-517.

Address for correspondence Ryunosuke Fukushi, MD, Department of Orthopedic Surgery, Sapporo Medical University, S1W16, Sapporo, Hokkaido 060-8556, Japan (e-mail: ryunosuke_fukushi_521@yahoo.co.jp).

\begin{abstract}
Keywords

- spinal process splitting laminectomy

- surgical invasiveness

- clinical results

- microendoscopic muscle-preserving interlaminar decompression

Study Design A retrospective comparative study.

Objective To compare retrospectively the clinical results and surgical invasiveness of two different types of minimally invasive surgery for lumbar spinal canal stenosis: microendoscopic muscle-preserving interlaminar decompression (ME-MILD) and spinal process splitting laminectomy (SPSL).

Summary of Background Data ME-MILD and SPSL are minimally invasive procedures. However, the two procedures have not been compared in the literature.

Materials and Methods We retrospectively enrolled patients who underwent MEMILD or SPSL from 2011 to 2015. The surgical invasiveness of each technique was determined by evaluating the time required for the surgical procedure, amount of blood loss, serum creatine kinase (CK) levels on postoperative day (POD) 1, C-reactive protein (CRP) levels on POD 3 and 7, and the hospitalization. The clinical results were evaluated using the Japanese Orthopaedic Association Back Pain Evaluation Questionnaire score, the Short Form (36) Health Survey patient-reported outcome score, the visual analog scale for pain, a patient satisfaction score, and the incidence of surgical complications.

Results A total of 97 patients were evaluated: 58 patients underwent ME-MILD, and 39 patients underwent SPSL. No significant differences were observed in the clinical results between the two groups. Regarding surgical invasiveness, no significant difference was found in the amount of blood loss, levels of CK, hospitalization, or time required for the procedure. However, CRP levels were significantly lower in the ME-MILD group.

Conclusions ME-MILD and SPSL are both minimally invasive procedures. In a comparison of these two procedures, CRP was significantly lower in the ME-MILD group.
\end{abstract}

\section{Introduction}

Lumbar spinal stenosis is a common spinal disease often treated surgically. Numerous posterior decompression procedures have been performed including wide laminectomy and fenestration. However, these conventional procedures damage posterior lumbar structures such as the lamina, facet joints, ligaments, and paravertebral muscles, sometimes resulting in postoperative lower back pain and intervertebral instability. ${ }^{1-3}$ Thus minimally invasive procedures are being utilized more frequently to prevent surgical damage to the posterior structures. ${ }^{4}$ In 2006, Mikami et $\mathrm{al}^{4}$ described a microendoscopic posterior decompression procedure called microendoscopy- received

April 7, 2017

accepted after revision

August 24, 2017

published online

January 2, 2018 (c) 2018 Georg Thieme Verlag KG Stuttgart · New York
DOI https://doi.org/ 10.1055/s-0037-1608871. ISSN 2193-6315. 
assisted muscle-preserving interlaminar decompression (MEMILD), with reference to Shiraishi's central cervical approach. ${ }^{5}$ In this technique, decompression is performed through a midline approach; thus invasiveness to the paravertebral muscles and facet joints can be minimized. In 2011, Watanabe et $\mathrm{al}^{6}$ described a spinous process splitting laminectomy (SPSL), in which the lamina is exposed by splitting the spinous process longitudinally into halves. The central approach in this technique also results in minimal invasiveness to the paravertebral muscles and facet joints. Moreover, SPSL can be performed without the assistance of an optical system such as a microendoscope or a microscope, resulting in a surgeon-friendly and affordable surgery.

Although favorable clinical results, including less surgical invasiveness, were reported previously for both ME-MILD and SPSL, these procedures have not been directly compared in any study. Therefore, we aimed to compare the clinical results and surgical invasiveness of ME-MILD and SPSL, clarifying the advantages and disadvantages of these two somewhat different minimally invasive surgeries.

\section{Materials and Methods}

\section{Patient Selection}

Patients with neurogenic claudication caused by lumbar spinal stenosis were considered candidates for surgery if conservative therapies (prostaglandin E1 with analgesics, epidural block, or selective nerve root block) administered for at least 3 months were ineffective. Patients with grade I degenerative lumbar spondylolisthesis without intervertebral instability were also considered for surgery. Intervertebral instability was defined as a sagittal translation $>3 \mathrm{~mm}$, segmental motion $>20$ degrees, or a posterior opening $>5$ degrees on flexion/extension radiographs. The inclusion criteria for the current study were previous decompression of one or two intervertebral levels with at least 6 months of follow-up. The exclusion criteria were a previous lumbar surgery and the presence of specific spinal disorders (infection, neoplasm, congenital diseases, or metabolic diseases). The surgical procedure was selected by surgeons according to their preferences; one surgeon possessing microendoscopic surgical skills selected ME-MILD, and the other five surgeons selected SPSL. All patient data were collected; the current study is a retrospective review of these data.

\section{Surgical Procedures}

\section{ME-MILD}

ME-MILD procedures were performed according to the methods described in Mikami and in Yoshimoto et al. ${ }^{4,7,8}$ For a onelevel (L4-L5) decompression, a posterior midline skin incision was made between the center of the $L 4$ spinous process and the cranial edge of the L5 spinous process to expose the tip of the caudal half of the L4 spinous process. The caudal half of the L4 spinous process was excavated using an air drill, preserving the periosteum of the lateral aspects of the spinous process. The interspinous ligament between L4 and L5 was split longitudinally in the midline, and the ligamentum flavum was exposed.

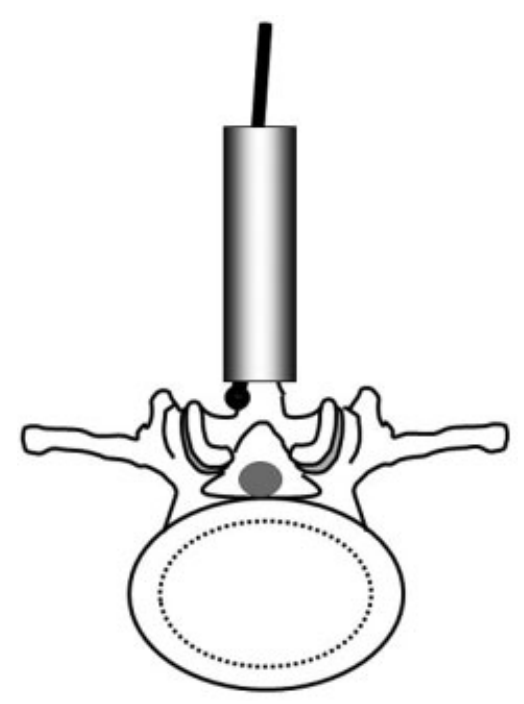

Fig. 1 Diagram for microendoscopic muscle-preserving interlaminar decompression surgical techniques. The caudal half of the $L 4$ spinous process was excavated and a tubular retractor was positioned in the midline. Microendoscopic decompression was performed in a symmetrical operative field.

A tubular retractor was positioned in the midline, and microendoscopic decompression was performed in a symmetrical operative field (-Fig. 1). Two-level surgery was performed with one additional skin incision.

SPSL

SPSL was performed according to the methods described by Watanabe et al. ${ }^{6}$ For a one-level (L4-L5) decompression, a posterior midline skin incision was made between the L3 and L5 spinous processes to expose the tip of the L4 spinous process. Using an osteotome, the L4 spinous process was divided bilaterally to the base and detached from the L4 lamina, leaving the bilateral paravertebral muscles attached to the split spinous process. The interspinous ligaments were also split longitudinally, and the two halves of the spinous process were retracted bilaterally. Decompression was performed in a symmetrical operative field (-Fig. 2). After the decompression, the two halves of the split L4 spinous process were sutured together using a strong surgical suture.

\section{Clinical Outcomes}

The Japanese Orthopaedic Association Back Pain Evaluation Questionnaire (JOABPEQ), normalized Short Form (36) Health Survey (SF-36), and visual analog scales (VAS; 0$100 \mathrm{~mm}$ ) were used to assess lower back pain, leg pain, and leg numbness before and after surgery. In addition, a patient satisfaction score (very satisfied, satisfied, dissatisfied, and very dissatisfied) was obtained before and after surgery, and surgical complications were evaluated.

Improvement in the JOABPEQ score was calculated as follows: postoperative score/preoperative score. The efficacy rate in terms of the JOABPEQ was calculated as follows: patients with a (1) postoperative score/preoperative score $\geq 20$ or (2) postoperative score $\geq 90$ and a preoperative score $<90$ were regarded as having obtained "efficacy," 


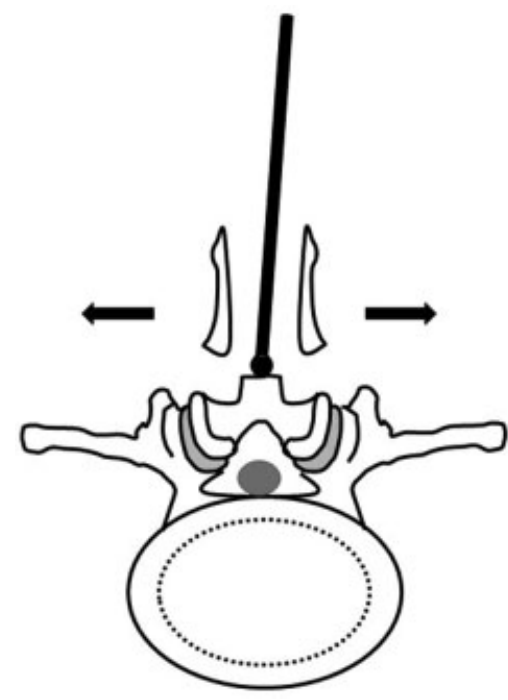

Fig. 2 Diagram for spinal process splitting laminectomy surgical techniques. The $L 4$ spinous process was divided bilaterally to the base and detached from the L4 lamina, leaving the bilateral paravertebral muscles attached to the split spinous process. Decompression was performed in a symmetrical operative field.

and the number of such patients was divided by the total number of patients in each group.

\section{Surgical Invasiveness}

Surgical invasiveness was evaluated using the operative time, blood loss, serum creatine kinase (CK) level on postoperative day (POD) 1, and C-reactive protein (CRP) level on POD 3 and 7.

\section{Imaging Evaluation}

The surgical invasiveness to the paravertebral muscles was assessed in patients who had pre- and postoperative magnetic resonance imaging (MRI). Postoperative fat infiltration into the multifidus muscles was assessed and compared with that in the preoperative images. On T1-weighted and T2-weighted axial MRI, high signal intensity regions located within the decompression level were regarded as fatty tissue. Fatty infiltration was diagnosed if the hyperintense regions were expanded in the postoperative images.

The MRI scanner used was a spine coil with GE Signa HDx 1.5 T (GE Healthcare, Milwaukee, Wisconsin, United States). The MRI parameters were as follows: T1-weighted image: TR/TE, 620/9.4 ms; receiver band width (RBW), \pm 31.25 $\mathrm{kHz}$; field of view (FOV), $15 \mathrm{~cm}$; matrix, $224 \times 192$; slice thickness/gap, $4 \mathrm{~mm} / 1 \mathrm{~mm}$; number of excitations (NEX), 2; total scan time, 3 minutes, 12 seconds; T2-weighted image: TR/TE, $4,000 / 102 \mathrm{~ms} ; \mathrm{RBW}, \pm 31.25 \mathrm{kHz} ; \mathrm{FOV}$, $15 \mathrm{~cm}$; matrix, $288 \times 192$; slice thickness/gap, $4 \mathrm{~mm} /$ $1 \mathrm{~mm}$; NEX, 4; total scan time, 3 minutes, 11 seconds.

\section{Statistical Analysis}

Continuous variables were evaluated using Student $t$ tests, discontinuous variables were evaluated using Mann-Whitney
Table 1 Clinical and demographic characteristics

\begin{tabular}{|l|l|l|l|}
\hline & ME-MILD & SPSL & $p$ value \\
\hline No. of patients & 58 & 39 & \\
\hline Age at surgery, $\mathrm{y}$ & $72.4 \pm 7.72$ & $73.1 \pm 10.8$ & 0.437 \\
\hline Sex, male/female & $34 / 24$ & $19 / 20$ & 0.337 \\
\hline Height, cm & $159.4 \pm 11.0$ & $155.3 \pm 9.7$ & 0.076 \\
\hline Weight, kg & $61.5 \pm 12.4$ & $61.8 \pm 12.1$ & 0.863 \\
\hline BMI, kg/m & $28.0 \pm 3.13$ & $25.2 \pm 3.49$ & 0.172 \\
\hline $\begin{array}{l}\text { No. of } \\
\text { decompression } \\
\text { levels }\end{array}$ & $1.54 \pm 0.41$ & $1.41 \pm 0.50$ & 0.385 \\
\hline $\begin{array}{l}\text { No. of patients } \\
\text { with } \\
\text { spondylolisthesis } \\
\text { (\%) }\end{array}$ & $13(22.4)$ & $8(20.5)$ & 0.842 \\
\hline
\end{tabular}

Abbreviation: BMI, body mass index; ME-MILD, microendoscopic muscle-preserving interlaminar decompression; SPSL, spinal process splitting laminectomy.

Note: Age, sex, height, weight, BMI, number of decompression levels, and number of patients with preoperative spondylolisthesis were similar between the two groups.

$U$ tests, and categorical variables were evaluated using chisquare tests. A $p$ value $<0.05$ was considered statistically significant.

\section{Ethics}

This study was approved by the institutional review board. Written informed consent was obtained from all patients before participation.

\section{Results}

\section{Follow-up Status}

A total of 137 patients underwent ME-MILD or SPSL between 2011 and 2015. Of these, 40 patients discontinued their scheduled clinic visits or did not respond to our letters. The remaining 97 patients (70.8\%) were successfully followed up $>6$ months and were thus enrolled in the current study. The mean postoperative follow-up period was 42.0 months (range: 6-59 months). Overall, 58 and 39 patients underwent ME-MILD and SPSL, respectively. - Table 1 summarizes the patient demographics and clinical data. There were no significant group differences in age, sex, height, weight, body mass index, number of decompression levels, or number of patients with preoperative spondylolisthesis.

\section{Clinical Outcomes}

No significant group differences were found in the JOABPEQ SF-36, VAS, or patient satisfaction scores, both pre- and postoperatively. In addition, the groups did not differ in complication rate; only two patients in the ME-MILD group required short-term antibiotic therapy for superficial surgical site infections. The clinical outcomes are summarized in -Tables 2-4 and -Figs. 3-8. 
514 ME-MILD versus Spinal Process Splitting Laminectomy Fukushi et al.

Table 2 Improvements in Japanese Orthopaedic Association Back Pain Evaluation Questionnaire score (postoperative score minus preoperative score)

\begin{tabular}{|l|l|l|l|}
\hline & ME-MILD & SPSL & $p$ value \\
\hline Low back pain & $24.2 \pm 37.3$ & $9.51 \pm 57.6$ & 0.676 \\
\hline Lumbar function & $8.03 \pm 36.9$ & $5.01 \pm 49.6$ & 0.910 \\
\hline Walking ability & $18.7 \pm 34.9$ & $9.66 \pm 53.9$ & 0.348 \\
\hline Social life function & $14.7 \pm 32.4$ & $7.66 \pm 26.6$ & 0.438 \\
\hline Mental health & $8.81 \pm 20.2$ & $9.1 \pm 40.4$ & 0.869 \\
\hline
\end{tabular}

Abbreviations: ME-MILD, microendoscopic muscle-preserving interlaminar decompression; SPSL, spinal process splitting laminectomy. Note: No significant group differences were observed.

Table 3 Efficacy rate in Japanese Orthopaedic Association Back Pain Evaluation Questionnaire

\begin{tabular}{|l|l|l|l|}
\hline & ME-MILD & SPSL & $p$ value \\
\hline Low back pain, \% & 59.2 & 66.6 & 0.472 \\
\hline Lumbar function, \% & 44.4 & 50.0 & 0.528 \\
\hline Walking ability, \% & 59.2 & 50.0 & 0.581 \\
\hline Social life function, \% & 44.4 & 33.3 & 0.472 \\
\hline Mental health, \% & 33.3 & 50.0 & 0.348 \\
\hline
\end{tabular}

Abbreviations: ME-MILD, microendoscopic muscle-preserving interlaminar decompression; SPSL, spinal process splitting laminectomy. Note: There were no significant group differences.

Table 4 Complication rates

\begin{tabular}{|l|l|}
\hline ME-MILD (\%) & $2(3.4)$ \\
\hline SPSL (\%) & $0(0)$ \\
\hline
\end{tabular}

Abbreviations: ME-MILD, microendoscopic muscle-preserving interlaminar decompression; SPSL, spinal process splitting laminectomy. Note: There were no significant group differences.

\section{Surgical Invasiveness}

No significant group differences were found in operative time, blood loss, or serum CK level on POD 1. However, the CRP levels on POD 3 and 7 were significantly lower in the ME-MILD group than in the SPSL group (POD 3, $2.20 \pm 2.50 \mathrm{mg} / \mathrm{dL}$ and $3.13 \pm 2.86 \mathrm{mg} / \mathrm{dL}$, respectively; POD $7,1.37 \pm 2.64 \mathrm{mg} / \mathrm{dL}$ and $1.55 \pm 1.44 \mathrm{mg} / \mathrm{dL}$, respectively) (-Table 5). This group difference among cases requiring two-level decompression and requiring one-level decompression are summarized in -Tables 6 and 7.

\section{Imaging Evaluation}

In the ME-MILD group, 12 patients (20.7\%) underwent an MRI examination both before and after surgery; in the SPSL group, there were 8 such patients (20.5\%). Patients underwent scanning at a mean of 18.2 months (range: 6-36 months) postoperatively in the ME-MILD group and 23.4 months (range: 6-51 months) postoperatively in the SPSL

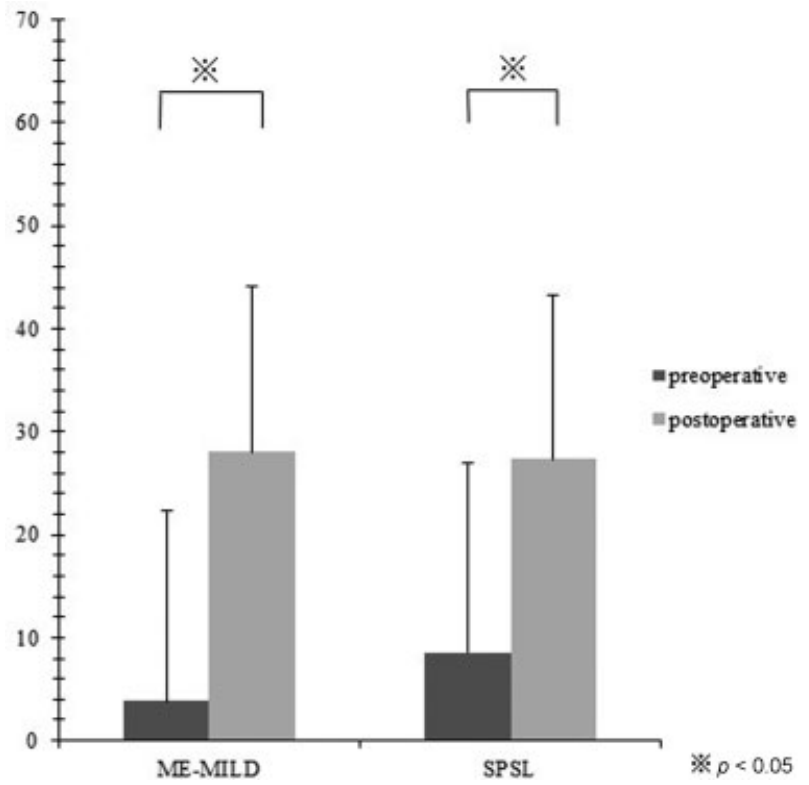

Fig. 3 Short Form (36) Health Survey Physical Component Summary scores. The mean preoperative (black) and postoperative (gray) scores are graphed. Significant improvement was noted in scores after surgery in both groups, but no significant group differences were observed in the pre- and postoperative periods. *Significant difference, $p<0.05$. ME-MILD, microendoscopic muscle-preserving interlaminar decompression; SPSL, spinal process splitting laminectomy.

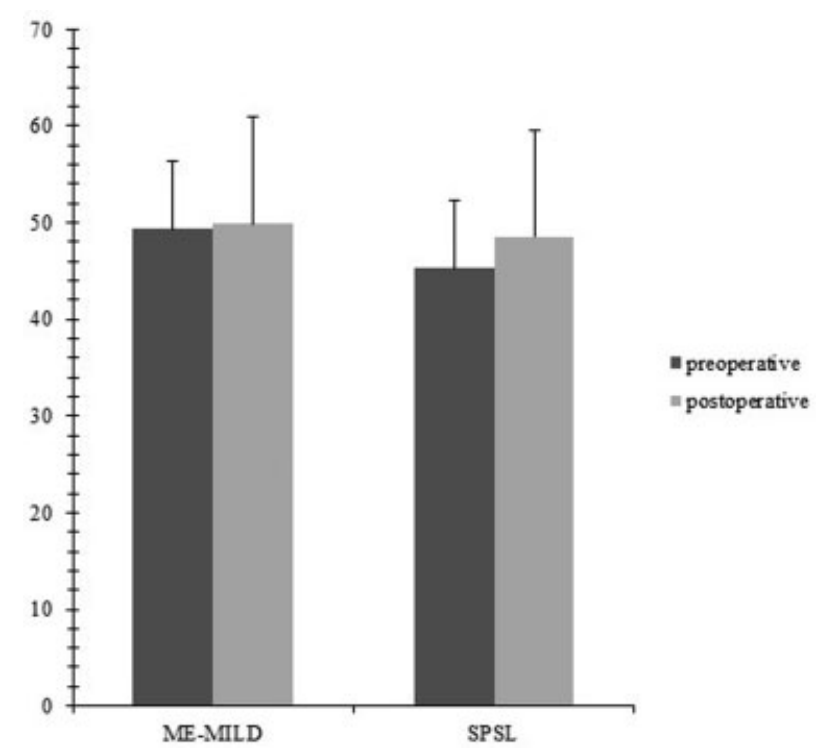

Fig. 4 Short Form (36) Health Survey Mental Component Summary scores. The mean preoperative (black) and postoperative (gray) scores are graphed. No significant group differences were found in the pre- and postoperative periods. ME-MILD, microendoscopic muscle-preserving interlaminar decompression; SPSL, spinal process splitting laminectomy.

group. Fat infiltration of the multifidus muscles was not observed in any patient. Representative MRI images from patients who underwent ME-MILD and SPSL are shown in -Figs. 9 and 10, respectively. In both cases, the spinal canal was fully decompressed, and there was no visible postoperative fat infiltration into the multifidus muscles. 


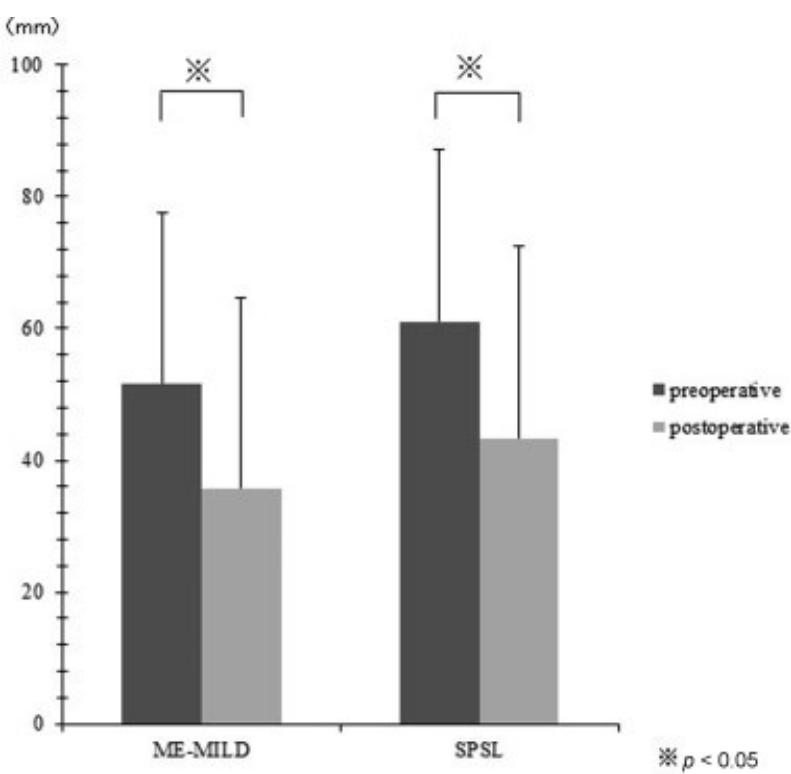

Fig. 5 Visual analog scale back pain scores. The mean preoperative (black) and postoperative (gray) scores are graphed. There was a significant improvement in back pain scores after surgery in both groups, but no significant group differences were noted in the pre- and postoperative periods. *Significant difference, $p<0.05$. ME-MILD, microendoscopic muscle-preserving interlaminar decompression; SPSL, spinal process splitting laminectomy.

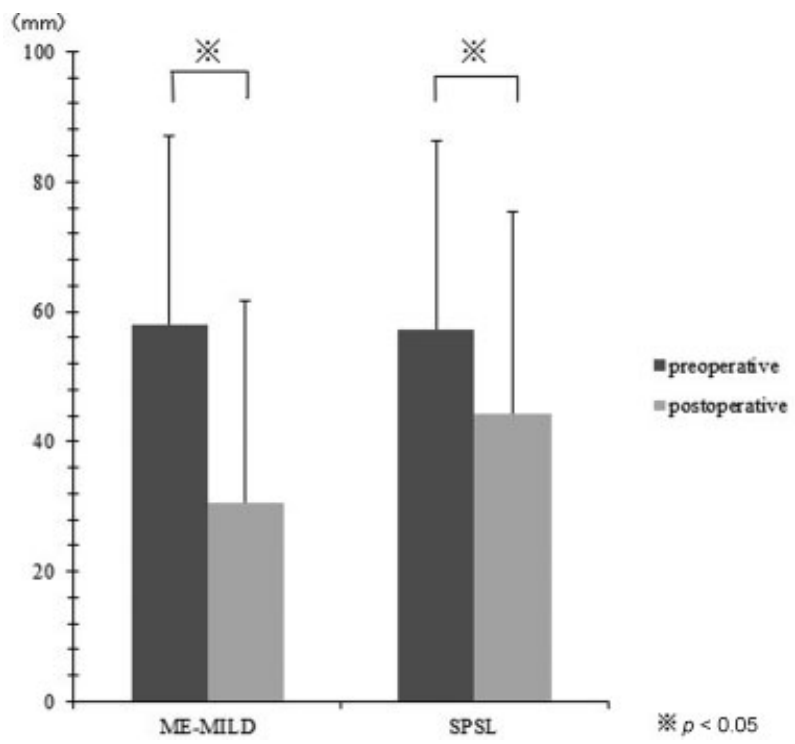

Fig. 6 Visual analog scale leg pain scores. The mean preoperative (black) and postoperative (gray) leg pain scores are graphed. There was a significant improvement in leg pain scores after surgery in both groups, but no significant group differences were observed in the preand postoperative periods. *Significant difference, $p<0.05$. MEMILD, microendoscopic muscle-preserving interlaminar decompression; SPSL, spinal process splitting laminectomy.

\section{Discussion}

Verbiest and Holland first described wide laminectomy for lumbar spinal stenosis in 1954. Since then, several posterior decompression procedures have been performed; however, conventional procedures are highly invasive and damage posterior lumbar structures. In particular, extensive invasion

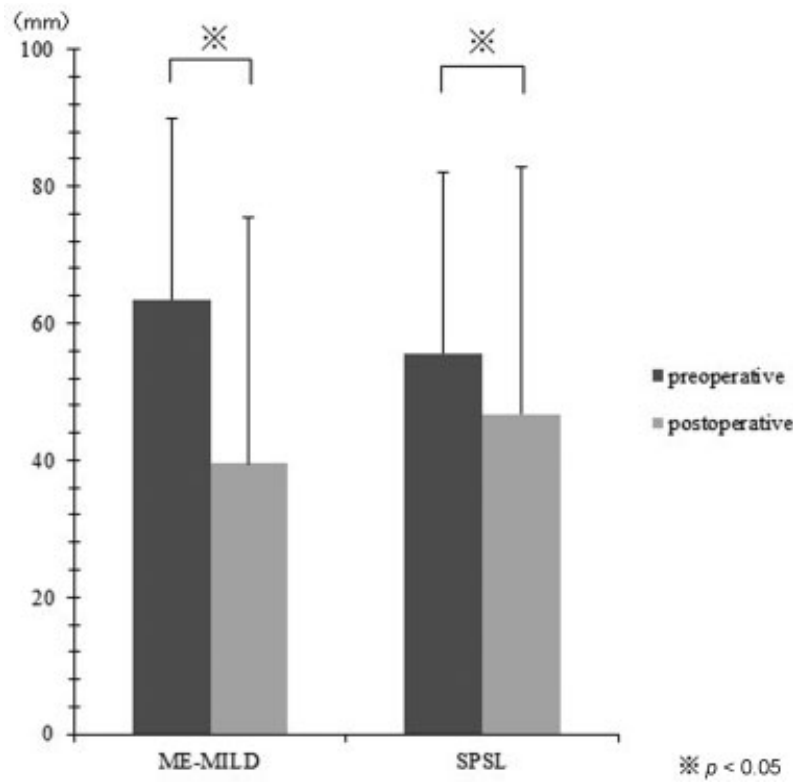

Fig. 7 Visual analog scale leg numbness scores. The mean preoperative (black) and postoperative (gray) leg numbness scores are graphed. There was a significant improvement in leg numbness scores after surgery in both groups, but there were no significant group differences in the pre- and postoperative periods. *Significant difference, $p<0.05$. ME-MILD, microendoscopic muscle-preserving interlaminar decompression; SPSL, spinal process splitting laminectomy.

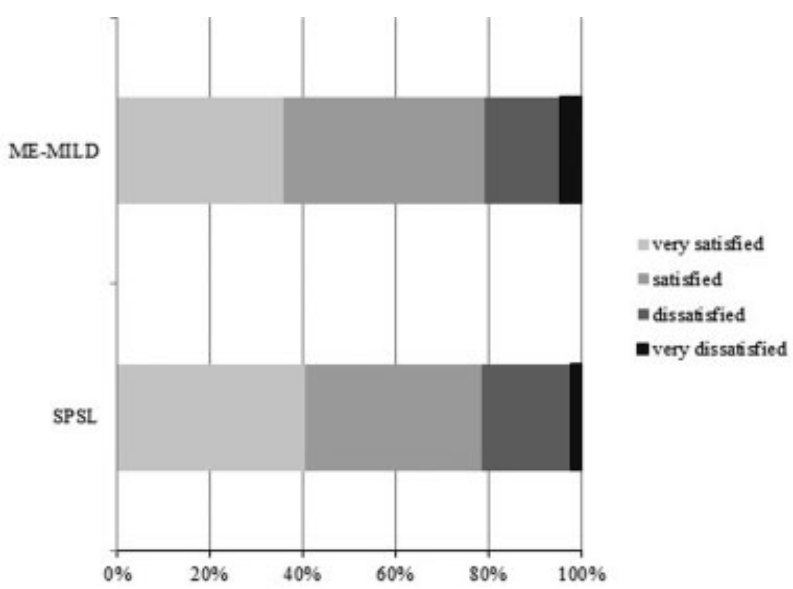

Fig. 8 Efficacy rate in terms of patient satisfaction scores. There were no significant group differences. ME-MILD, microendoscopic musclepreserving interlaminar decompression; SPSL, spinal process splitting laminectomy.

of the paravertebral muscles and facet joints often results in atrophy of the paravertebral muscles, postoperative lower back pain, and intervertebral instability. ${ }^{1-3}$

ME-MILD and SPSL use a similar concept to reduce surgical invasiveness, namely that a central approach more easily preserves the facet joints and the attachment sites of the paravertebral muscles to the spinous process. Furthermore, the use of microendoscopy in ME-MILD minimizes the length of the skin incision (15-20 mm). However, ME-MILD requires surgeons to be highly skilled in microendoscopic surgery. In contrast, SPSL does not require microendoscopic surgical skills and is thus a surgeon-friendly procedure, but it 
Table 5 Surgical invasiveness

\begin{tabular}{|l|l|l|l|}
\hline & ME-MILD & SPSL & $p$ value \\
\hline & $(\boldsymbol{n}=58)$ & $(\boldsymbol{n}=39)$ & \\
\hline $\begin{array}{l}\text { Operative time, } \\
\text { min }\end{array}$ & $111.0 \pm 39.2$ & $102.5 \pm 38.6$ & 0.121 \\
\hline $\begin{array}{l}\text { Intraoperative } \\
\text { blood loss, mL }\end{array}$ & $17.9 \pm 30.8$ & $14.3 \pm 24.1$ & 0.586 \\
\hline CK POD 1, IU/L & $142 \pm 171.4$ & $110 \pm 93.6$ & 0.058 \\
\hline $\begin{array}{l}\text { CRP POD 3, } \\
\text { mg/dL }\end{array}$ & $2.20 \pm 2.50$ & $3.13 \pm 2.86$ & $0.027^{\text {a }}$ \\
\hline $\begin{array}{l}\text { CRP POD 7, } \\
\mathrm{mg} / \mathrm{dL}\end{array}$ & $1.37 \pm 2.64$ & $1.55 \pm 1.44$ & $0.013^{\mathrm{a}}$ \\
\hline $\begin{array}{l}\text { Hospitalization, } \\
\mathrm{d}\end{array}$ & $28.8 \pm 33.3$ & $25.6 \pm 9.84$ & 0.282 \\
\hline
\end{tabular}

Abbreviations: CK, creatine kinase; CRP, C-reactive protein; ME-MILD, microendoscopic muscle-preserving interlaminar decompression; POD, postoperative day; SPSL, spinal process splitting laminectomy.

Note: No significant group differences were found in operative time, blood loss, serum CK level on POD 1, or hospitalization. However, the CRP level on POD 3 and 7 were significantly lower in the ME-MILD group than in the SPSL group.

${ }^{a}$ Significant difference, $p<0.05$.

Table 6 Surgical invasiveness among cases requiring laminectomy for one intervertebral level

\begin{tabular}{|l|l|l|l|}
\hline & ME-MILD & SPSL & $p$ value \\
\hline & $(\boldsymbol{n}=\mathbf{2 9})$ & $(\boldsymbol{n}=\mathbf{2 3})$ & \\
\hline $\begin{array}{l}\text { Operative time, } \\
\text { min }\end{array}$ & $84.6 \pm 28.2$ & $83.4 \pm 30.3$ & 0.111 \\
\hline $\begin{array}{l}\text { Intraoperative } \\
\text { blood loss, mL }\end{array}$ & $8.6 \pm 17.1$ & $12.6 \pm 20.9$ & 0.813 \\
\hline CK POD 1, IU/L & $142 \pm 115.4$ & $112 \pm 102.6$ & 0.085 \\
\hline $\begin{array}{l}\text { CRP POD 3, } \\
\text { mg/dL }\end{array}$ & $2.11 \pm 2.62$ & $2.92 \pm 2.37$ & 0.052 \\
\hline $\begin{array}{l}\text { CRP POD 7, } \\
\text { mg/dL }\end{array}$ & $1.09 \pm 1.84$ & $1.53 \pm 1.28$ & $0.023^{\text {a }}$ \\
\hline $\begin{array}{l}\text { Hospitalization, } \\
\text { d }\end{array}$ & $24.1 \pm 14.1$ & $26.3 \pm 11.6$ & 0.284 \\
\hline
\end{tabular}

Abbreviations: CK, creatine kinase; CRP, C-reactive protein; ME-MILD, microendoscopic muscle-preserving interlaminar decompression; POD, postoperative day; SPSL, spinal process splitting laminectomy. Note: The CRP level on POD 7 was significantly lower in the ME-MILD group than in the SPSL group.

${ }^{a}$ Significant difference, $p<0.05$.

requires a relatively long skin incision. In the current study, we directly compared the clinical outcomes and surgical invasiveness of these two procedures.

Regarding the clinical outcomes, no significant differences were found between the two procedures, and the outcomes were comparable with those previously reported for conventional procedures. For example, Phan and Mobbs ${ }^{9}$ and den Boogert et $\mathrm{al}^{10}$ reported satisfaction rates of $74.5 \%$ and $69.1 \%$, respectively, for conventional fenestration, similar to the satis-
Table 7 Surgical invasiveness among cases requiring laminectomy for two intervertebral levels

\begin{tabular}{|l|l|l|l|}
\hline & ME-MILD & SPSL & $p$ value \\
\hline & $(\boldsymbol{n}=\mathbf{2 9 )}$ & $(\boldsymbol{n}=\mathbf{1 6})$ & \\
\hline $\begin{array}{l}\text { Operative time, } \\
\text { min }\end{array}$ & $139.7 \pm 26.5$ & $133.3 \pm 31.5$ & 0.119 \\
\hline $\begin{array}{l}\text { Intraoperative } \\
\text { blood loss, mL }\end{array}$ & $27.2 \pm 38.2$ & $16.4 \pm 27.6$ & 0.338 \\
\hline CK POD 1, IU/L & $140 \pm 215.6$ & $107 \pm 80.7$ & 0.056 \\
\hline $\begin{array}{l}\text { CRP POD 3, } \\
\text { mg/dL }\end{array}$ & $2.28 \pm 2.42$ & $3.35 \pm 3.42$ & $0.022^{\text {a }}$ \\
\hline $\begin{array}{l}\text { CRP POD 7, } \\
\text { mg/dL }\end{array}$ & $1.63 \pm 3.26$ & $1.52 \pm 1.64$ & 0.217 \\
\hline $\begin{array}{l}\text { Hospitalization, } \\
\text { d }\end{array}$ & $33.3 \pm 4.9$ & $26.1 \pm 7.1$ & 0.514 \\
\hline
\end{tabular}

Abbreviations: CK, creatine kinase; CRP, C-reactive protein; ME-MILD, microendoscopic muscle-preserving interlaminar decompression; POD, postoperative day; SPSL, spinal process splitting laminectomy.

Note: The CRP level on POD 3 was significantly lower in the ME-MILD group than in the SPSL group.

${ }^{\mathrm{a}}$ Significant difference, $p<0.05$.
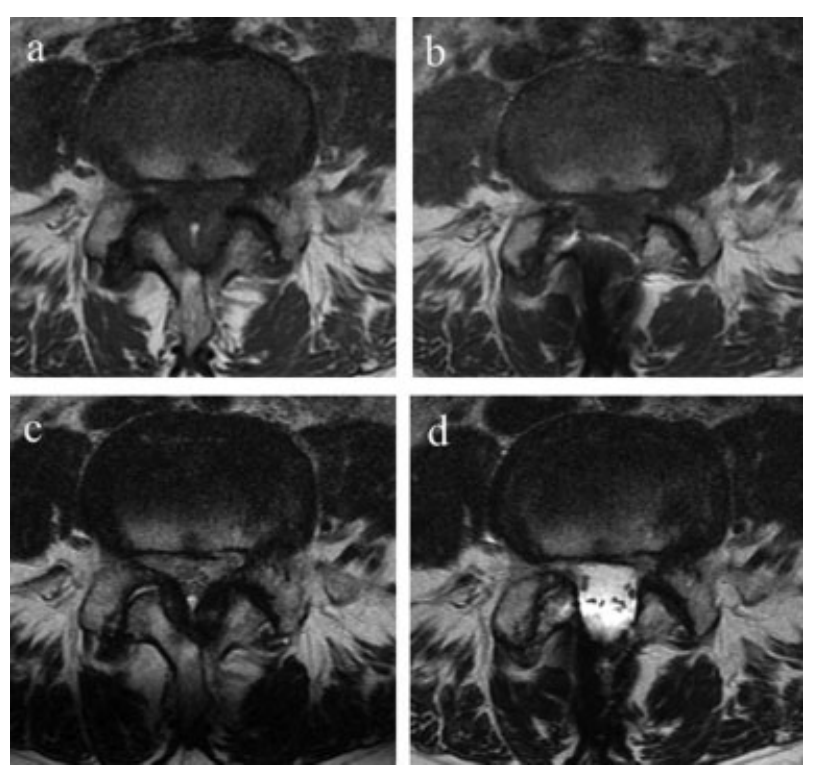

Fig. 9 Representative case of microendoscopic muscle-preserving interlaminar decompression. (a) Preoperative and (b) postoperative T1-weighted magnetic resonance imaging (MRI) and (c) preoperative and (d) postoperative T2-weight MRI showed successful spinal canal decompression and no significant changes of the multifidus muscles.

faction rate in the current study. Regarding surgical invasiveness, both procedures showed less invasiveness compared with that previously reported for classical fenestration. For example, Watanabe et $\mathrm{al}^{6}$ reported a CRP level on POD 3 of $5.5 \mathrm{mg} / \mathrm{dL}$ and intraoperative bleeding of $55 \mathrm{~mL}$, and Hashidate and Takahashi $^{11}$ reported a serum CK level on POD 1 of 295 IU/L, a CRP level on POD 3 of $2.43 \mathrm{mg} / \mathrm{dL}$, and intraoperative bleeding of $49 \mathrm{~mL}$ for classical fenestration. In contrast, in the current study, serum CK level on POD 1 was $141 \mathrm{IU} / \mathrm{L}$, CRP level on POD 3 was $2.20 \mathrm{mg} / \mathrm{dL}$, 

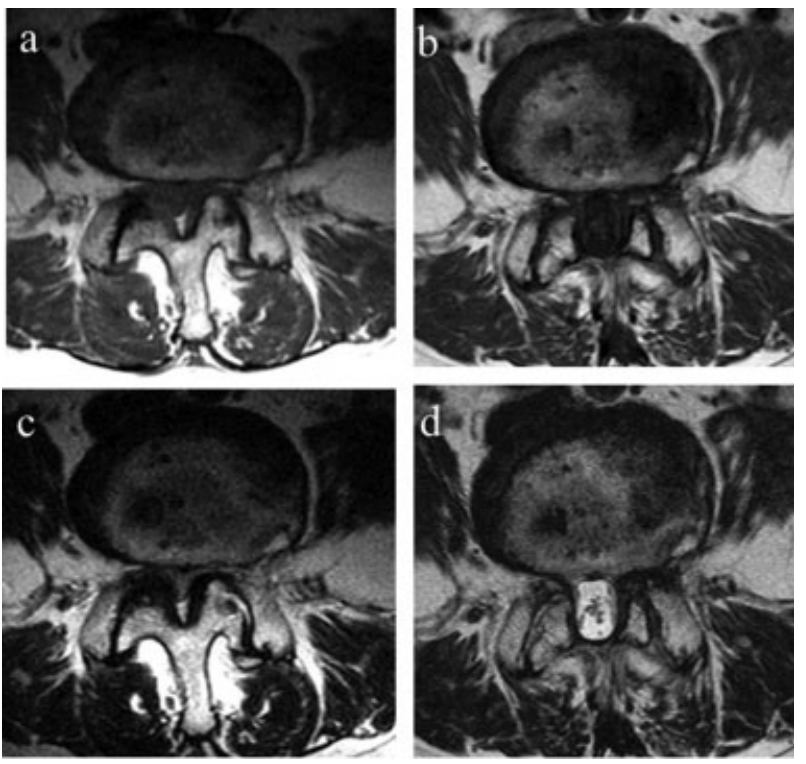

Fig. 10 Representative case of spinal process splitting laminectomy. (a) Preoperative and (b) postoperative T1-weighted magnetic resonance imaging (MRI) and (c) preoperative and (d) postoperative T2-weighted MRI showed successful spinal canal decompression and no significant changes of the multifidus muscles.

intraoperative bleeding was $17 \mathrm{~mL}$ for the ME-MILD group, and was $110 \mathrm{IU} / \mathrm{L}, 3.10 \mathrm{mg} / \mathrm{dL}$, and $14 \mathrm{~mL}$, respectively, for the SPSL group. Thus both ME-MILD and SPSL offer reduced invasiveness and good clinical results.

In a direct comparison, the advantage of ME-MILD over SPSL was limited to obtaining slightly lower CRP levels on POD 3 and 7 , even though it is a technically demanding procedure with a steep learning curve and requires an expensive optical system. For surgeons with microendoscopic surgical skills, ME-MILD can minimize surgical invasiveness without compromising reliability or prolonging operative time. However, compared with ME-MILD, SPSL requires fewer special skills, such as not needing a tubular retractor. It is therefore considered a relatively easy operation for young novice surgeons. In this study, we found similar clinical results between the groups. Thus when a surgeon has little surgical experience, SPSL is ideal because it is considered relatively easy. Additionally, for most surgeons, SPSL is a surgeon-friendly procedure with good clinical results and relatively low invasiveness.

We attempted to evaluate the fat infiltration of the multifidus muscle due to invasion after surgery on the MRI. Our imaging evaluation did not reveal postoperative fatty infiltration of the multifidus muscles, suggesting that both ME-MILD and SPSL are minimally invasive to the paravertebral muscles. However, there was a large variation in the length of time between the surgery and the postoperative MRI. Fat infiltration of the multifidus muscles was also evaluated visually, rather than quantitatively. Furthermore, only a few patients in either group underwent MRI postoperatively. Future research with a larger sample size, standardized length of time for follow-up, and quantitative evaluation of the MRI images will be necessary.

The current study has several limitations including its retrospective design, small sample sizes, different group sizes, short follow-up duration, and relatively low followup rate. The patients included were often referrals from general practice clinics. After the surgery, the patients often requested that postoperative examinations and rehabilitation be performed at the referring clinic. We believe this is why the follow-up rate in this study was low. Furthermore, the most significant limitation may be that the procedure selection (ME-MILD or SPSL) depended on the preference of each surgeon. In the ME-MILD group, one surgeon familiar with the procedure performed the surgeries; in the SPSL group, five spinal surgeons performed the surgeries. However, all five spinal surgeons operating on patients in the SPSL group are experienced specialists and have performed numerous similar spinal surgeries in their careers as surgeons. Therefore, we believe there were no major differences in surgical ability among these five surgeons. Thus we think this had a limited effect on the results of the study.

\section{References}

1 Herno A, Airaksinen O, Saari T. Long-term results of surgical treatment of lumbar spinal stenosis. Spine 1993;18(11):1471-1474

2 Lee CK. Lumbar spinal instability (olisthesis) after extensive posterior spinal decompression. Spine 1983;8(04):429-433

3 Sihvonen T, Herno A, Paljärvi L, Airaksinen O, Partanen J, Tapaninaho A. Local denervation atrophy of paraspinal muscles in postoperative failed back syndrome. Spine 1993;18(05):575-581

4 Mikami Y, Nagae M, Ikeda T, Tonomura H, Fujiwara H, Kubo T. Tubular surgery with the assistance of endoscopic surgery via midline approach for lumbar spinal canal stenosis: a technical note. Eur Spine J 2013;22(09):2105-2112

5 Shiraishi T. A new technique for exposure of the cervical spine laminae. Technical note. J Neurosurg 2002;96(1, Suppl):122-126

6 Watanabe K, Matsumoto M, Ikegami T, et al. Reduced postoperative wound pain after lumbar spinous process-splitting laminectomy for lumbar canal stenosis: a randomized controlled study. J Neurosurg Spine 2011;14(01):51-58

7 Yoshimoto M, Miyakawa T, Takebayashi T, et al. Microendoscopyassisted muscle-preserving interlaminar decompression for lumber spinal stenosis: clinical results of consecutive 105 cases with more than 3-year follow-up. Spine (Phila Pa 1976) 2014;39:E318-E325

8 Yoshimoto M, Takebayashi T, Kawaguchi S, et al. Minimally invasive technique for decompression of lumbar foraminal stenosis using a spinal microendoscope: technical note. Minim Invasive Neurosurg 2011;54(03):142-146

9 Phan K, Mobbs RJ. Minimally invasive versus open laminectomy for lumbar stenosis: A systematic review and meta-analysis. Spine 2016;41(02):E91-E100

10 den Boogert HF, Keers JC, Marinus Oterdoom DL, Kuijlen JM. Bilateral versus unilateral interlaminar approach for bilateral decompression in patients with single-level degenerative lumbar spinal stenosis: a multicenter retrospective study of 175 patients on postoperative pain, functional disability, and patient satisfaction. J Neurosurg Spine 2015;23(03):326-335

11 Hashidate H, Takahashi J, Nakamura I, et al. Short-term results of laminectomy preserving paraspinal muscles for lumbar canal stenosis. Central Jpn J Orthopaed Surg Traumato 2007;50(05):883-884 